\title{
MMP-2 downregulation mediates differential regulation of cell death via ErbB-2 in glioma xenografts
}

\author{
CHRISTOPHER S. GONDI ${ }^{1}$, DZUNG H. DINH ${ }^{2}$, JEFFREY D. KLOPFENSTEIN ${ }^{2}$, \\ MEENA GUJRATI $^{3}$ and JASTI S. RAO ${ }^{1,2}$
}

Departments of ${ }^{1}$ Cancer Biology and Pharmacology, ${ }^{2}$ Neurosurgery, ${ }^{3}$ Pathology, University of Illinois College of Medicine at Peoria, Peoria, IL, USA

Received February 24, 2009; Accepted April 29, 2009

DOI: 10.3892/ijo_00000335

\begin{abstract}
The epidermal growth factor receptor (EGFR) family (also known as the ErbB protein family) is comprised of four structurally-related receptor tyrosine kinases. Insufficient ErbB signaling in humans is associated with the development of neurodegenerative diseases, such as multiple sclerosis and Alzheimer's disease. In contrast, excessive ErbB signaling is associated with the development of a wide variety of solid tumors. ErbB-1 and -2 are found in many human cancers and their excessive signaling may be critical factors in the development and malignancy of solid tumors. Several molecular strategies have been developed recently to modulate either EGFR or the downstream signal beyond the cell surface receptor. In the present study, we used human EGFR-overexpressing glioma xenograft cells 4910 and 5310 and targeted MMP-2 expression using an adenoviral RNAi construct. We observed that the RNAi-mediated downregulation of MMP-2 causes the upregulation of ErbB-2 in certain EGFR-overexpressing glioma xenograft cells both in vitro and in vivo. Targeted MMP-2 downregulation was observed in a dosedependent manner with no apparent off-target effects in these xenograft cells. We also noted that the overexpression of ErbB-2 induced by MMP-2 downregulation is consistent with p50-mediated cell death in 5310 cells but not in 4910 cells. In addition, APAF-1 expression levels increased in correlation with increased ErbB-2 expression after MMP-2 downregulation in vitro and in vivo. Our results suggest that MMP-2 may play a role in a hitherto unknown signaling pathway mediated via ErbB-2 in certain cancer cell types.
\end{abstract}

\section{Introduction}

The metastasis of cancer cells involves multiple steps, which include detachment from the tumor mass and invasion of the surrounding extracellular matrix (ECM) (1). This invasion process is accompanied by the degradation of both macro-

Correspondence to: Dr J.S. Rao, Department of Cancer Biology and Pharmacology, University of Illinois College of Medicine at Peoria, One Illini Drive, Peoria, IL 61605, USA

E-mail: jsrao@uic.edu

Key words: MMP-2, cell death, ErbB-2, glioma molecular components of the ECM and the basement membrane and is regulated by the intrinsic properties of tumor cells as well as microenvironmental factors (2). Matrix metalloproteinases (MMPs) comprise a family of zincdependent endopeptidases that are capable of degrading components of the ECM (3). MMPs have been implicated in normal matrix remodeling events [e.g., tissue and organ development, wound healing (4)] and abnormal matrix remodeling (5) events [e.g., tumor invasion and metastasis (6)]. High levels of MMP-2 and -9 have been found to correlate with enhanced metastasis and poor prognosis in patients with breast and other cancers (7). Furthermore, MMP-2 activity is associated with the risk for a relapse in breast cancer patients (8).

The ErbB family of receptor kinases includes four closely related members: epidermal growth factor receptor (EGFR or ErbB-1), $-2,-3$ and -4 (9). Among the various family members, ErbB-2 is most directly related to breast cancer, and its relevance has recently been recognized in glioma patients (10). Amplification of ErbB-2 is found in $20-30 \%$ of cancer patients who have solid tumors associated with poor prognosis (8).

Epidermal growth factor (EGF)-like proteins comprise a group of structurally similar growth factors, which contain a conserved six-cysteine residue motif called the EGF-domain (11). EGF-like factors are synthesized as transmembrane precursors. These precursors can undergo proteolytic cleavage at the cell surface to release a mature soluble ectodomain, a process often referred to as 'ectodomain shedding'. Ectodomain shedding of EGF-like factors has been linked to MMPs. Once shed from the cell surface, EGF-like factors bind to ErbB-1, $-2,-3$ or $-4(12,13)$. Heterodimerization or homodimerization of these receptors following ligand binding drives intracellular signal transduction cascades, which in turn, results in diverse cell fates including proliferation, differentiation, migration and inhibition of apoptosis (12). In addition to its role in driving normal developmental processes, studies show that de-regulated ErbB signaling is associated with tumor formation in a variety of tissues and that ectodomain shedding of EGFlike factors is a critical event in this process. Although no specific ligand has yet been identified for ErbB-2, it is the preferred heterodimerization partner for all ErbB family members, and it plays a pivotal role in intracellular signaling mediated by other ErbB receptors. The status of ErbB-2 
expression in a given cell is critical in determining cellular response to growth factors or environmental stimuli. Thus, knowledge of the molecular mechanisms by which EGF-like factors are shed from the cell surface and the nature of the proteases that governs this process are crucial in understanding ErbB receptor signaling and, ultimately, the development of novel cancer therapeutics that target the ErbB pathway.

\section{Materials and methods}

Construction of hpRNA-expressing adenovirus. The adenovirus was constructed using an adenoviral pSuppressor kit (Imgenex) as previously described (14). The pSuppressor plasmid (pSup-3) containing the MMP-2 siRNA sequence 5'-AACGGACAAAGAGTTGGCAGTATCGATACTGCC AACTCTTTGTCCGTT-3' was digested with $P a c I$ and cotransfected with pAd vector backbone in 293 cells to generate an adenovirus containing MMP-2 siRNA (Ad-MMP-2). The pSV construct was used to construct an adenovirus containing the scrambled sequence (Ad-SV). Adenovirus generation, amplification, and titer were completed as previously described (15). Viruses were plaque purified, propagated on 293 cells, and purified by cesium chloride gradient according to standard techniques. Particle titers of all adenoviruses were determined by absorbance measurements at $260 \mathrm{~nm}$, and functional titers (plaque-forming units) were determined by end-point dilution titration in 293 cells. The amount of infective adenoviral vector per cell (plaque-forming units/cell) in culture medium was expressed as multiplicity of infection (MOI).

Cell lines and culture conditions. 4910 and 5310 human glioma xenograft cells were maintained as a monolayer in DMEM/F12 medium supplemented with 10\% FBS, 50 units/ml penicillin and $50 \mu \mathrm{g} / \mathrm{ml}$ streptomycin (Life Technologies Inc., Frederick, MD) at $37^{\circ} \mathrm{C}$ in a humidified $5 \% \mathrm{CO}_{2}$ atmosphere.

Gelatin zymography. MMP activity in conditioned medium was determined by gelatinase zymography as described previously (16). 4910 and 5310 human glioma xenograft cells were infected with mock, Ad-SV, and the indicated doses of Ad-MMP-2 for $36 \mathrm{~h}$. The cells were washed and incubated overnight with serum-free medium. Conditioned medium containing equal amounts of protein were electrophoresed in $7.5 \%$ SDS-polyacrylamide gels containing $1.5 \mathrm{mg} / \mathrm{ml}$ gelatin. The gels were washed and gently shaken in three consecutive washings in $2.5 \%$ Triton $\mathrm{X}-100$ solution to remove SDS. The gels were then incubated at $37^{\circ} \mathrm{C}$ overnight in incubation buffer [50 mmol/l Tris- $\mathrm{HCl}$ (pH 7.5), 0.05\% $\mathrm{NaN}_{3}, 5 \mathrm{mmol} / \mathrm{C} \mathrm{CaCl}_{2}$, and $1 \mu \mathrm{mol} / 1 \mathrm{ZnCl}_{2}$. The gel was stained with $0.1 \%$ Coomassie brilliant blue in $10 \%$ acetic acid and $10 \%$ isopropanol and subsequently destained for $1 \mathrm{~h}$. Gelatinolytic activities were identified as clear zones of lysis against a dark blue background.

Western blot analysis. 4910 and 5310 human glioma xenograft cells were infected with mock, Ad-SV, and the indicated doses of Ad-MMP-2 for $36 \mathrm{~h}$. Cells were collected and total cell lysates were prepared in standard RIPA extraction buffer containing aprotinin and phenyl-methyl-sulfonyl-fluoride. Twenty micrograms of protein from these samples were separated under non-reducing conditions by $12 \%$ SDS-PAGE and transferred to nitrocellulose membranes (Schleicher \& Schuell, Keene, NH). The membranes were immunoprobed for $2 \mathrm{~h}$ with antibodies against p50, APAF-1, ErbB-2, -3, -4, and caspase- 8 as per standard protocols. Then, membranes were immunoprobed with appropriate HRP-conjugated secondary antibody, and developed according to enhanced chemiluminescence protocol (Amersham, Arlington Heights, IL). For loading control, the membranes were stripped and probed with monoclonal antibodies for GAPDH as per standard protocol.

Reverse transcription PCR. Total RNA was isolated from the control and Ad-MMP-2-infected cells using TRIzol according to standard protocol after $6,12,24,48$ and $72 \mathrm{~h}$ of infection. Total RNA was treated with DNase I (Invitrogen, Carlsbad, CA) to remove contaminating genomic DNA. First strand cDNA was prepared using Superscript III reverse transcriptase (Invitrogen). One hundred nanograms of first strand cDNA were used for PCR amplification, and the primers for MMP-2 GAPDH were used as the internal control. PCR analysis was performed as per standard protocol. MMP-2 mRNA expression was normalized to GAPDH and expressed as percent expression.

\begin{tabular}{llc} 
& \multicolumn{1}{c}{ Primers } & Position \\
MMP-2 & GTGCTGAAGGACACACTAAAGAAGA & $494-518$ \\
& CCTACAACTTTGAGAAGGATGGCAA & $1074-1098$ \\
GAPDH & GGAGTCAACGGATTTGGTCGTAT & $93-116$ \\
& GTCTTCACCACCATGGAGAAGGCT & $376-399$
\end{tabular}

FACS analysis. To analyze the impact of MMP-2 downregulation on glioma cell death, 4910 and 5310 human glioma xenograft cells were infected with various recombinant adenoviral constructs as described previously. After $36 \mathrm{~h}$ of infection, cells were harvested, stained with propidium iodide and sorted for sub-diploid DNA (apoptotic cell) content with a Becton-Dickinson FACS Calibur flow cytometer.

In vivo glioma studies. To determine the effect of MMP-2 downregulation in vivo, nude mice were implanted with 4910 or 5310 glioma xenograft cells. Briefly, nude mice were initially subcutaneously implanted with $\left(5 \times 10^{6}\right) 4910$ or 5310 human glioma cells and allowed to grow for 10 days. After subcutaneous tumor development, the subcutaneous tumors were harvested and finely cut followed by trypnisation in $1 / 10$ strength trypsin. After complete tissue disruption, the tumor suspension was washed 3 times by repeated centrifugation (1000 $\mathrm{g}$ for $3 \mathrm{~min}$ ) and resuspended in serum-free DMEM. The cells were then counted and intracranially injected $\left(1 \times 10^{6}\right.$ cells per mouse), followed by intracranial injection of Ad-MMP-2 virus $\left(1 \times 10^{6}\right.$ or $\left.10 \times 10^{6} \mathrm{pfu}\right)$ after 10 days of glioma implantation. After adenovirus administration, mice were further allowed to grow for 15 days after which they were euthanized and brains harvested. The brains were paraffin-embedded and sectioned as per standard protocols followed by immunostaining of ErbB-2 or APAF-1 with appropriate primary and HRP conjugated (for ErbB-2) or Texas red conjugated (for APAF-1) secondary antibodies followed by visualization by light or fluorescent microscopy. The animal experiments were carried out according to our university ethics guidelines. 

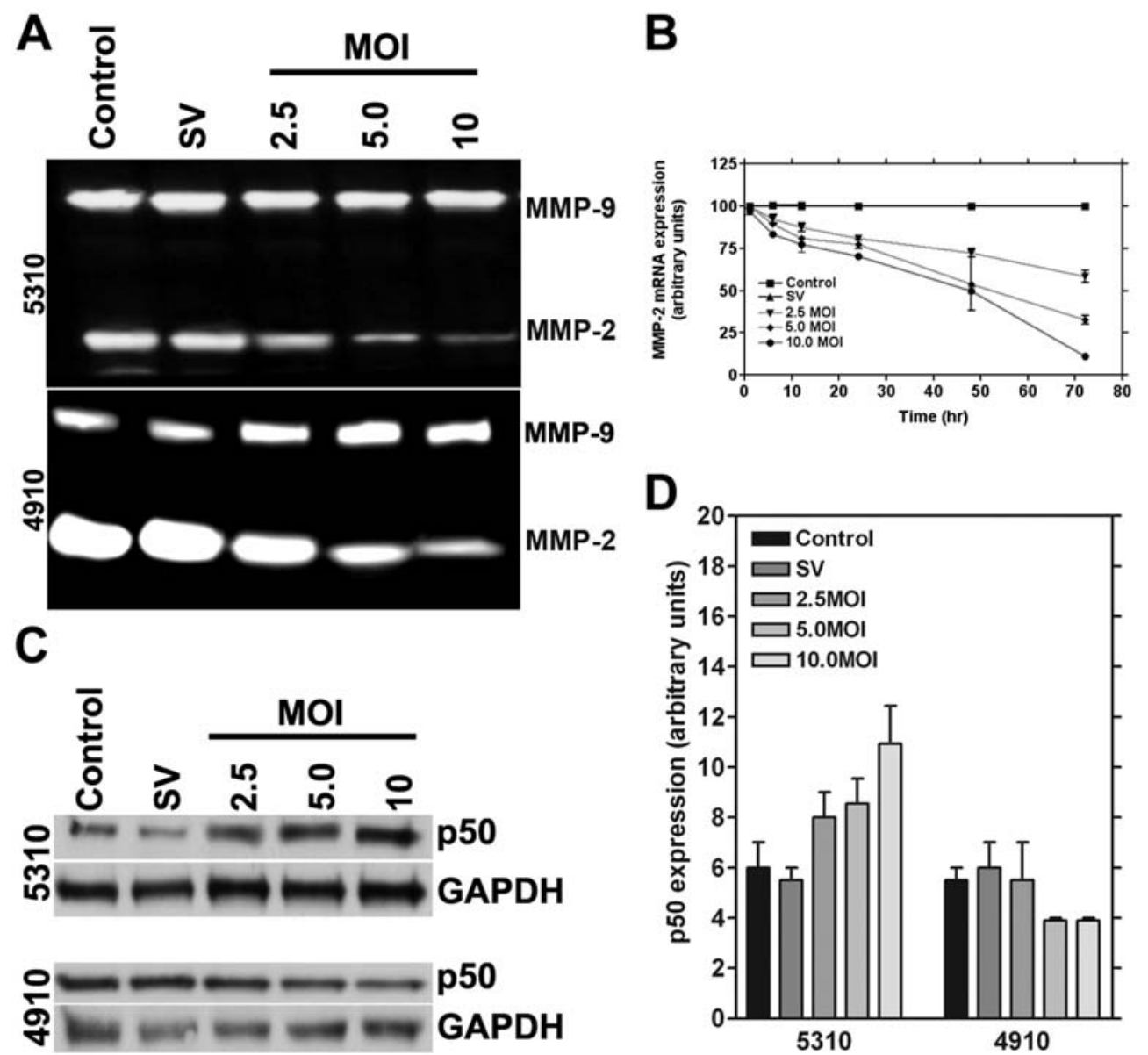

Figure 1. RNAi-mediated downregulation of MMP-2 causes reduction of MMP-2 enzymatic activity and MMP-2 mRNA expression in 4910 and 5310 human glioma xenograft cells and causes the upregulation of p50 in 5310 cells but not in 4910 cells. Human glioma xenograft cells 4910 and 5310 were infected with Ad-SV (scrambled vector) at 10 MOI or Ad-MMP-2 at 2.5, 5 or 10 MOI for $48 \mathrm{~h}$. MMP-2 activity in the conditioned medium was determined by gelatin zymography (A). Time course analysis of MMP-2 mRNA expression was also determined by RT-PCR analysis after 6, 12, 24, 48 and $72 \mathrm{~h}$ of adenoviral infection in 4910 or 5310 cells using 2.5, 5 and 10 MOI of Ad-MMP-2 (performed in triplicate) (B). For the control, we used uninfected or 10 MOI of Ad-SV. Expression levels of the p50 subunit of the NF-kB complex were determined by Western blot analysis (C). Expression levels of GAPDH were also determined and served as loading controls. Quantitative analysis of p50 expression levels was carried out with densitometry, and the experiments were performed in triplicate (D).

\section{Results}

RNAi-mediated downregulation of MMP-2 causes decreased MMP-2 expression and activity in 4910 and 5310 human glioma xenograft cells. Glioma cells are known to produce high levels of MMP-2 and -9, which account for their invasive behavior. To determine the effect of MMP-2 downregulation in glioma xenograft cells, we used RNAi-based methods to construct an adenovirus targeting MMP-2. From the gelatin zymography results, we observed that RNAi-mediated downregulation of MMP-2 was specific and had no off-target effects (Fig. 1A). To determine whether RNAi-mediated downregulation of MMP-2 changes over time, we carried out realtime PCR analysis for MMP-2 mRNA levels after infection with 2.5, 5.0 and 10.0 MOI of Ad-MMP-2 at 6, 12, 24, 48 and $72 \mathrm{~h}$. We observed that the decrease in MMP-2 expression was dose-dependent and MMP-2 expression decreased over time in a non-linear manner (Fig. 1B).

RNAi-mediated downregulation of MMP-2 causes accumulation of p50 levels in 5310 glioma xenograft cells but not in 4910 glioma xenograft cells. It is known that NF- $\mathrm{KB}$ is associated with certain apoptotic events (17). NF- $\mathrm{\kappa B}$ is best characterized for its protective activity in response to proapoptotic stimuli; its role in suppressing programmed necrosis has come to light more recently. NF- $\kappa \mathrm{B}$ is a complex composed of p50 and p65 subunits. To determine the role of p50 in MMP-2 regulation, we downregulated MMP-2 as described earlier in Materials and methods. Western blot analysis for p50 was carried out in glioma xenograft cells that were downregulated for MMP-2. We observed that p50 levels increased in a dose-dependent manner in 5310 cells, but not in 4910 cells (Fig. 1C). Quantitative analysis confirmed that p50 levels increased with increasing MOI of Ad-MMP-2 in 5310 cells. In 4910 cells, p50 levels decreased with the maximum decrease observed at $5 \mathrm{MOI}$ of Ad-MMP-2 (Fig. 1D).

RNAi-mediated downregulation of MMP-2 causes APAF-1 accumulation in 5310 human glioma xenograft cells but not in 4910 cells. When mitochondria are exposed to apoptotic stimuli, cytochrome $\mathrm{c}$ is released from the mitochondria into the cytoplasm. This cytochrome $\mathrm{c}$ in the cytoplasm associates with APAF-1 in the presence of dATP or ATP, thereby 


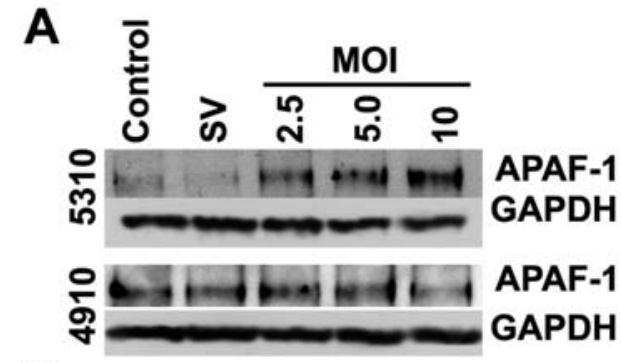

B

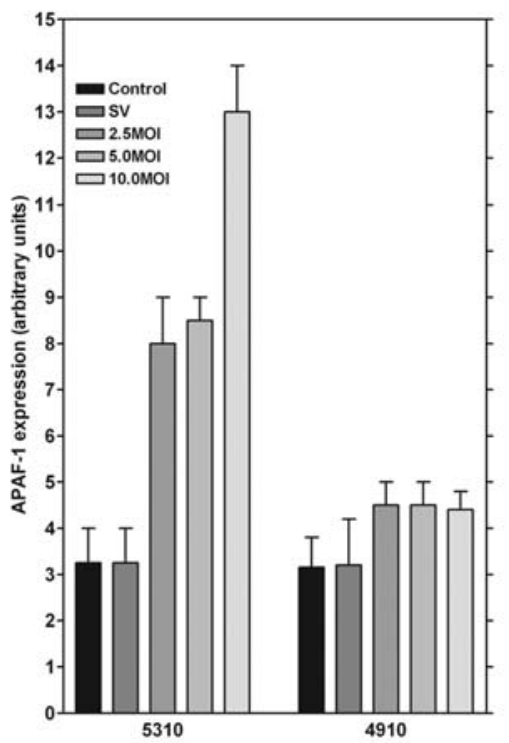

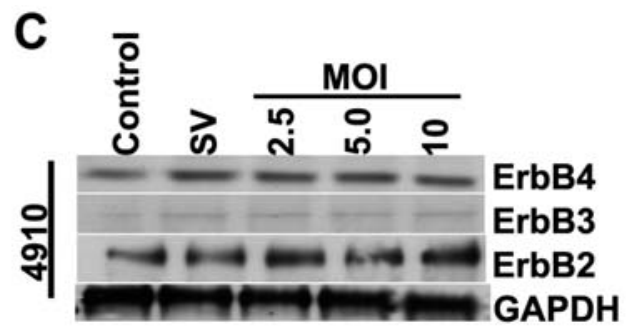

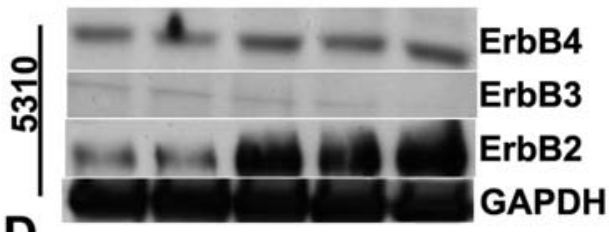

D

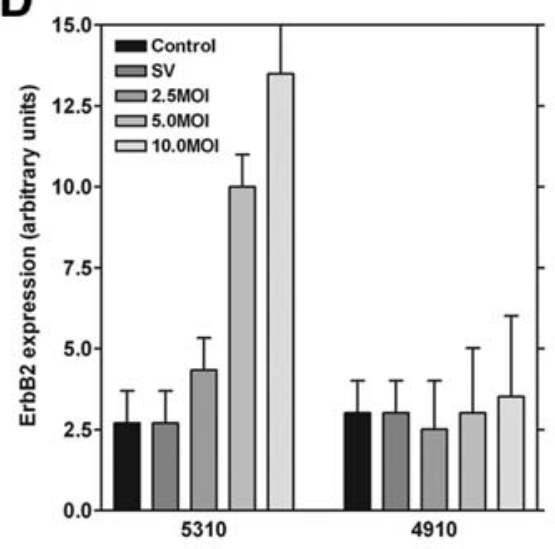

Figure 2. Downregulation of MMP-2 causes the upregulation of APAF-1 and ErbB-2 in 5310 but not in 4910 human glioma xenograft in vitro. Human glioma xenograft cells 4910 and 5310 were infected with Ad-SV at 10 MOI or Ad-MMP-2 at 2.5, 5 or 10 MOI for 48 h. APAF-1 expression levels were determined by Western blot analysis. Expression levels of GAPDH were also determined and served as loading controls (A). Quantitative analysis of APAF-1 expression levels was determined by densitometry normalized to GAPDH expression levels. Experiments were performed in triplicate (B). Expression levels of ErbB-2, -3 , and -4 were determined by Western blot analysis (C). Expression levels of GAPDH were also determined and served as loading controls. Quantitative analysis of ErbB-2 expression levels was determined by densitometry normalized to GAPDH expression levels, and experiments were performed in triplicate (D).

forming the apoptosome and activating caspase-9 (18). To determine whether MMP-2 downregulation causes the activation of the apoptosome complex, we measured APAF-1 expression levels in 4910 and 5310 human glioma xenograft cells after treatment with varying MOI of Ad-MMP-2. In 5310 cells, we observed that APAF-1 levels increased in a dose-dependent manner whereas we observed no significant change in APAF-1 levels in 4910 cells (Fig. 2A). Quantitative analysis revealed a 4-fold increase in APAF-1 levels in 5310 cells, but no appreciable change in APAF-1 levels in 4910 cells (Fig. 2B). The in vivo studies confirmed the in vitro results; from the immunohistochemical studies we observed the accumulation of APAF-1 in Ad-MMP-2 injected mice implanted with 5310 human glioma xenografts but not with 4910 glioma xenografts. In both the cases reduction in tumor volume was observed in a dose-dependent manner when compared to controls (Fig. 2B).

RNAi-mediated downregulation of MMP-2 causes ErbB-2 accumulation in 5310 human glioma xenograft cell but not in 4910 cells. To determine the effect of MMP-2 downregulation on ErbB-2, -3 and -4 levels, Western blot analysis was performed to measure expression levels of ErbB-2, -3, and -4. The results indicated no appreciable change in the expression levels of ErbB-2, -3, or -4 in 4910 cells after Ad-MMP-2 infection, whereas ErbB-2 levels increased in a dose-dependent manner in 5310 cells (no change in ErbB-3 or -4 levels) (Fig. 2C). Quantitative analysis showed that ErbB-2 levels in 5310 cells increased 6-fold with 10 MOI of Ad-MMP-2; no significant change was observed in 4910 cells (Fig. 2D). Similar to APAF-1 expression the in vivo studies confirmed the in vitro results; from the immunohistochemical studies we observed the accumulation of ErbB-2 in Ad-MMP-2 injected mice implanted with 5310 human glioma xenografts but not with 4910 glioma xenografts. In both the cases reduction in tumor volume was observed in a dose-dependent manner when compared to controls (Fig. 3).

RNAi-mediated downregulation of MMP-2 causes activation of caspase-8 in 4910 human glioma xenograft cell but not in 5310 cells. To determine whether MMP-2 downregulation causes the activation of caspase-8, we assessed caspase- 8 activation in 4910 and 5310 human glioma xenograft cells after treatment with varying MOI of Ad-MMP-2. In 4910 cells, we observed that levels of cleaved caspase- 8 increased in a dose-dependent manner whereas no significant change in cleaved caspase- 8 was observed in 5310 cells (Fig. 4A).

RNAi-mediated downregulation of MMP-2 causes G1/GO arrest in 4910 and 5310 human glioma xenograft cells. To 


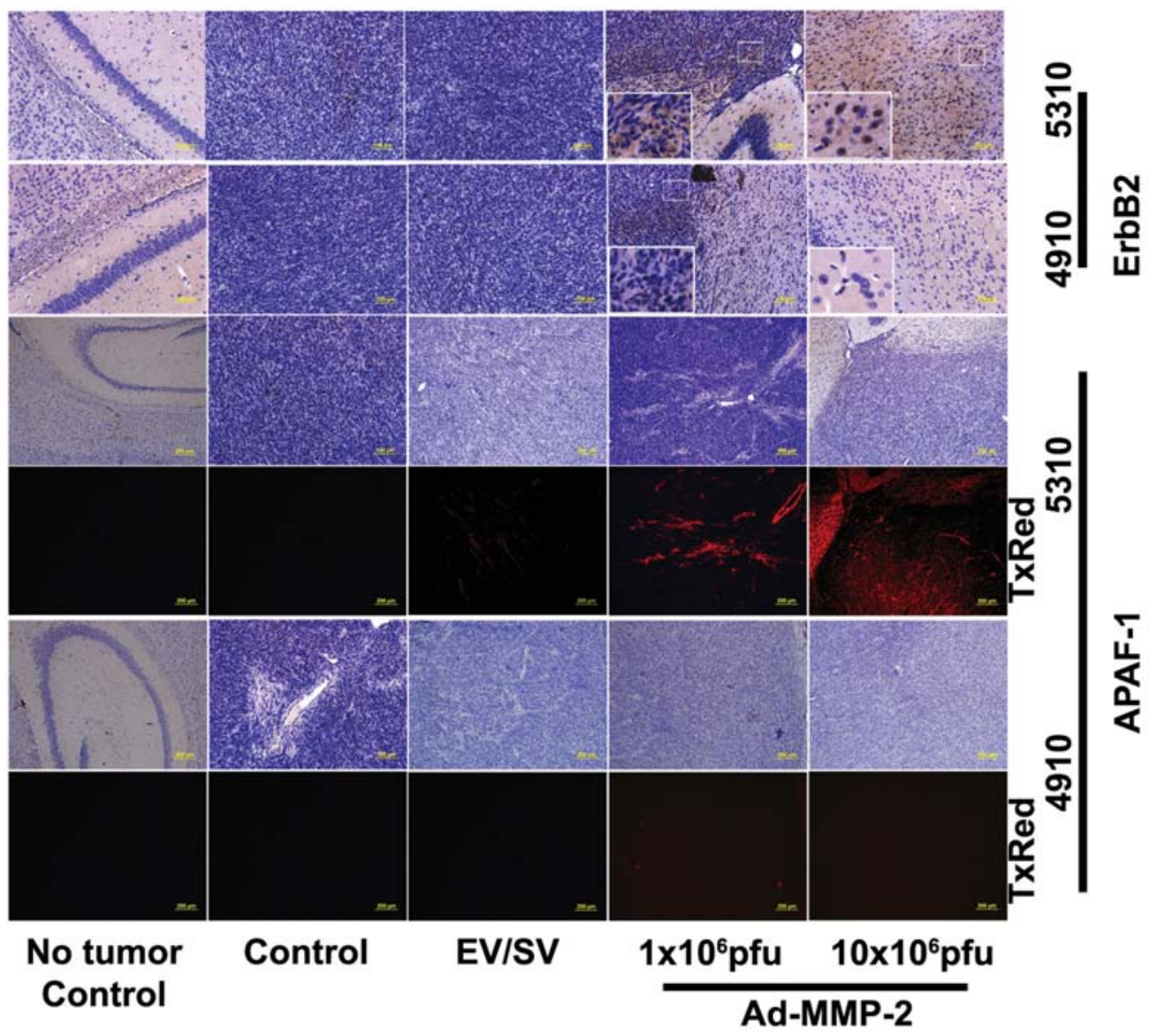

Figure 3. Downregulation of MMP-2 causes the upregulation of APAF-1 and ErbB-2 in 5310 but not in 4910 human glioma xenograft cells in vivo. Nude mice with pre-established intracranial human glioma tumors (4910 or 5310) were treated with Ad-MMP-2 by intracranial injections (1 or 10x10 6 pfu). Ten days following Ad-MMP-2 administration the brains were harvested sectioned and immunoprobed for ErbB-2 and APAF-1 using appropriate HRP conjugated (for ErbB-2) or TxRed conjugated (for APAF-1) secondary antibody.

determine whether MMP-2 downregulation causes cell cycle arrest, we used FACS analysis to assess DNA content of 4910 and 5310 human glioma xenograft cells after treatment with varying MOI of Ad-MMP-2. The FACS analysis results show dose-dependent arrest in G0/G1 phase in 5310 cells. However, in 4910 cells, the sub-G0 population increased significantly in a dose-dependent manner (Fig. 4B).

\section{Discussion}

Characteristics of human malignant glioma are excessive proliferation, infiltrative growth, angiogenesis, and suppression of anti-tumor immune surveillance. In this study, we examined the effect of MMP-2 downregulation in glioma xenograft cell lines 4910 and 5310. Gliomas are known to produce high levels of proteases, particularly MMP-9 and -2. From the gelatin zymography results, we observed that 4910 cells overexpress MMP-2 as compared to MMP-9; however, expression levels of MMP-2 and -9 were similar in 5310 cells. From our time course studies, we observed that downregulation of MMP-2 occurs in a dose- and time-dependent manner. MT1MMP activates pro-MMP-2 via its interaction with TIMP-2, which serves as an intermolecular bridge for pro-MMP-2 binding to MT-MMPs (19), hence, targeting MMPs would be useful in controlling glioma invasiveness.
Further, MMPs are known to be associated with cell surface molecules like CD44, which can cause intracellular signaling (20). Although the role of NF- $\mathrm{NB}$ in cell survival is well known, its role in the induction of apoptotic events is only now being understood. NF- $\mathrm{KB}$ most commonly antagonizes programmed cell death (PCD) by activating the expression of anti-apoptotic proteins and antioxidant molecules, but it can also promote PCD under certain conditions and in certain cell types (21). In our study, we observed that 5310 cells show increased expression of the p50 component of NF-кB whereas we did not observe any change in the case of 4910 cells. Researchers have also shown that MKP-1 is an NF-кB-mediated pro-survival effecter in attenuating JNK-mediated pro-apoptotic response (22). Western blot analysis revealed that APAF-1 levels increased in 5310 cells whereas no significant change was observed in 4910 cells. APAF-1 is involved in mitochondrial-mediated cell death where caspase-9, cytochrome $\mathrm{c}$ and the APAF-1 complex form the apoptosome complex. In mammalian cells, caspases-9, -8, and -2 rely on apoptosome complex, death-inducing signaling complex (DISC), and PIDDosome, respectively, for activation (23). In our study, as evidenced by FACS analysis, 4910 cells show caspase- 8 activation accompanied by DNA degradation, whereas APAF-1 upregulation is not accompanied by caspase- 8 activation in 


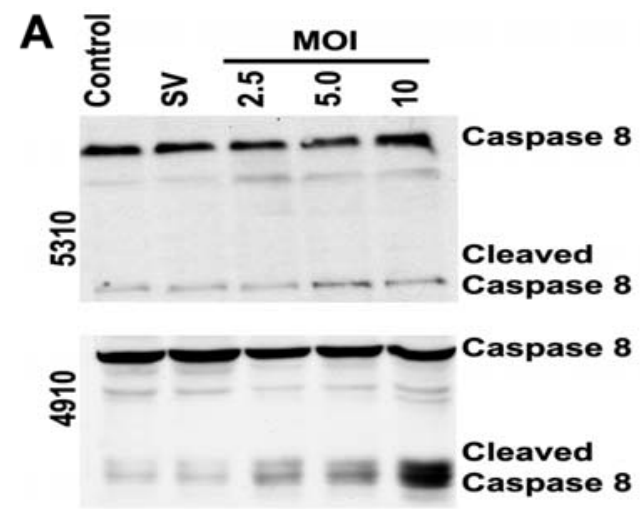

5310 cells. These results indicate that 4910 cells follow the DISC pathway and 5310 cells follow the apoptosome complex pathway.

In our previous studies, we demonstrated that MMP-2 downregulation induced apoptotic cell death in vitro and suppressed tumor growth of pre-established U-251 intracranial xenografts in nude mice (24). However, the actual mechanism and the signaling events by which apoptotic cell death occurs after MMP-2 regulation have not yet been identified. The present study gives an indication of the possible mechanisms by which these apoptotic events may

B

5310

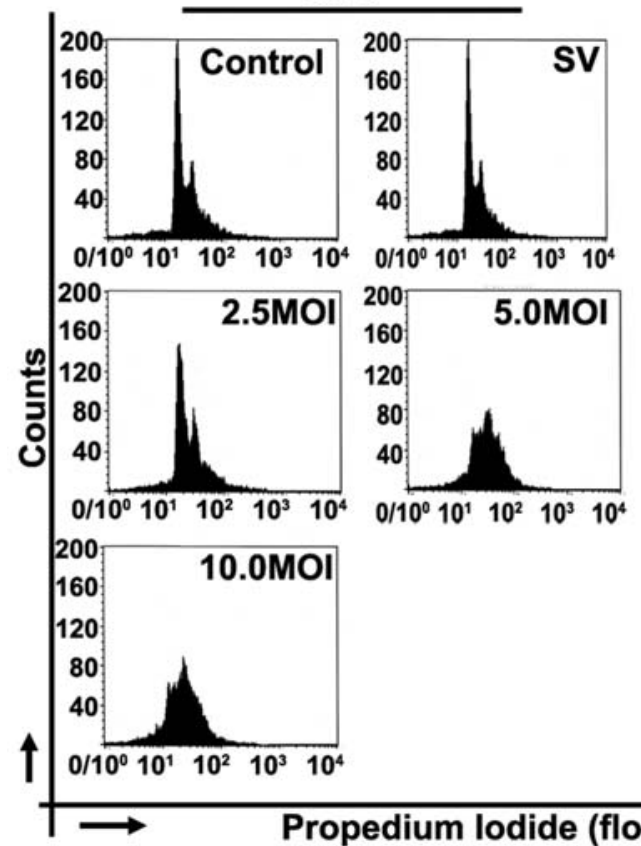

4910
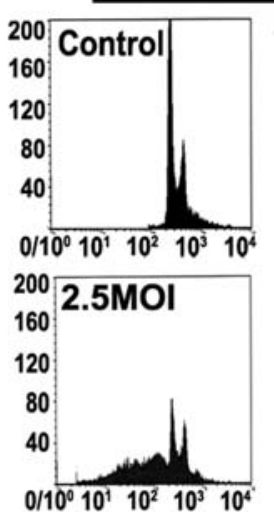

$0 / 10^{0} 10^{1} 10^{2} 10^{3} 10^{4}$

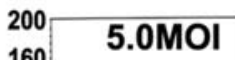

160

120

80

40

$0 / 10^{0} 10^{1} 10^{2} \quad 10^{3} 10^{4}$

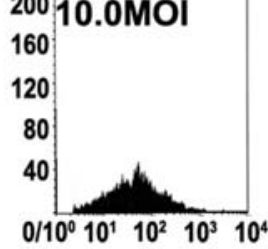

Figure 4. Downregulation of MMP-2 causes activation of caspase-8 in 4910 cells but not in 5310 human glioma xenograft cells, induces G0/G1 arrest in 5310 cells, and induces an increase in sub-G0 population in 4910 cells. Human glioma xenograft cells 4910 and 5310 were infected with Ad-SV at 10 MOI or AdMMP-2 at 2.5, 5 or $10 \mathrm{MOI}$ for $48 \mathrm{~h}$. Caspase-8 expression levels were determined by Western blot analysis (A). To determine initiation of apoptosis, cells were stained with propidium iodide after RNase treatment and sorted by FACS analysis. A total of 10000 cells were sorted per treatment condition (B).

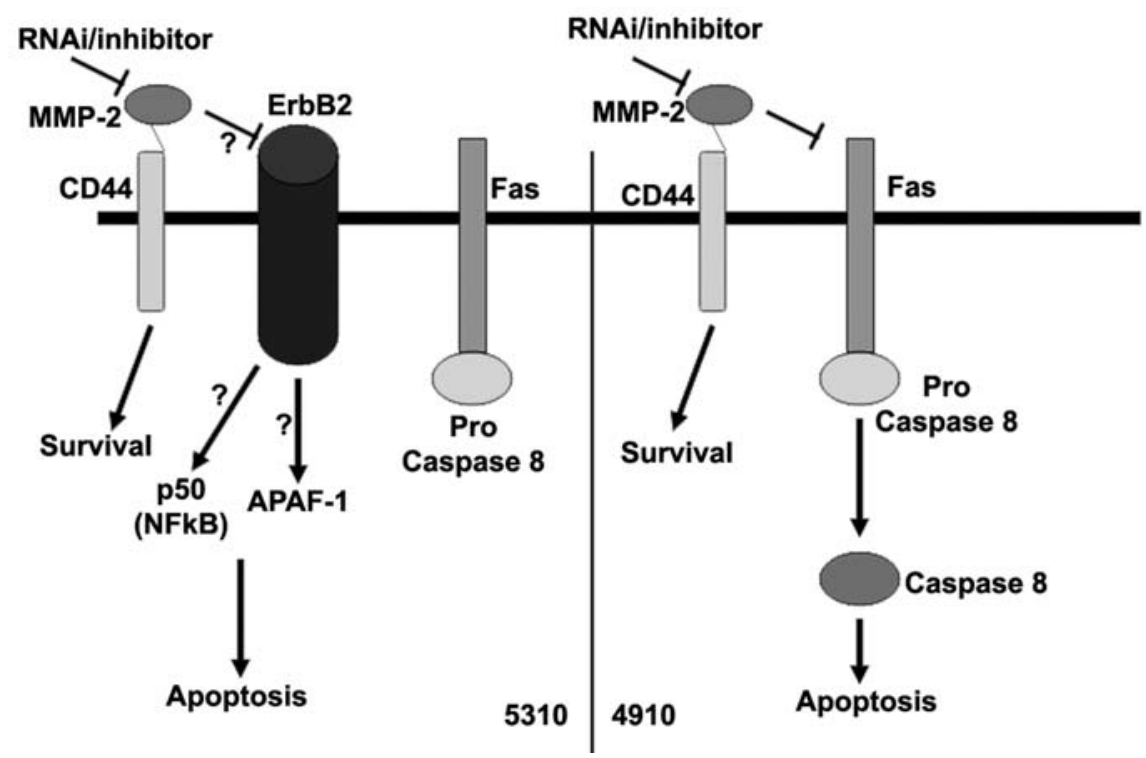

Figure 5. Schematic representation of plausible ErbB-2-mediated apoptotic pathway in 5310 glioma xenograft cells and Fas-mediated apoptotic pathway in 4910 glioma xenograft cells. 
take place. Interestingly, no DNA damage was detected in 5310 cells; the cells were arrested in the G0/G1 phase after MMP-2 downregulation. In contrast, the sub-G0 population of cells increased in 4910 cells, indicating DNA damage and formation of apoptotic bodies, which is further evidence for DISC-mediated apoptosis.

In the present study, we also observed that downregulation of MMP-2 causes an increase in levels of ErbB-2 in 5310 cells but not in 4910 cells. From the in vivo studies we observed similar results essentially confirming in vitro studies. ErbBs belong to the EGFR family and EGF-like factors are synthesized as transmembrane precursors. These precursors can undergo proteolytic cleavage at the cell surface to release a mature soluble ectodomain, a process referred to as 'ectodomain shedding'. Ectodomain shedding of EGF-like factors has been linked to MMPs and a disintegrin and metalloprotease (ADAM) families. Shedding can be activated by a variety of pharmacological and physiological stimuli and these activation events have been linked to the enhancement of metalloprotease activity, possibly via the action of intracellular signaling modules. Once shed from the cell surface, EGF-like factors bind to a family of four cell surface receptors named ErbB-1, -2, -3 and -4 (25). Our results demonstrate that MMP-2 may be responsible for accumulation of ErbB-2 in 5310 cells but not in 4910 cells. This accumulation may be mediated by the lack of ectodomain shedding as reported earlier (Fig. 5). Further studies on the dimerization of ErbBs may be necessary to determine the mode of intracellular signaling after MMP-2 downregulation. Researchers have demonstrated that overexpression of ErbB-2 is positively associated with elevated levels of MMP-2 and MMP-9 (26). In this study we demonstrate the negative regulation of ErbB-2 in 5310 cells in relation to MMP-2. Our results demonstrate the possibility of multiple roles for MMP-2 and ErbB-2 in apoptosis regulation. In both of these xenograft cell lines, inhibition of proliferation was achieved (data not shown). Taken together, these results demonstrate the therapeutic potential of targeting MMP-2. To our knowledge this is the first report indicating the involvement of MMP-2 with ErbB-2.

\section{Acknowledgements}

We thank Shellee Abraham for assistance in the manuscript preparation and Diana Meister and Sushma Jasti for manuscript review. This research was supported by National Cancer Institute Grant CA75557, CA92393, CA95058, CA116708, N.I.N.D.S. NS47699, NS57529, and NS61835 and Caterpillar, Inc., OSF St. Francis, Inc. Peoria, IL (to J.S.R.). The contents are solely the responsibility of the authors and do not necessarily represent the official views of NIH.

\section{References}

1. Herlyn M and Malkowicz SB: Regulatory pathways in tumor growth and invasion. Lab Invest 65: 262-271, 1991.

2. Bouterfa H, Darlapp AR, Klein E, Pietsch T, Roosen K and Tonn JC: Expression of different extracellular matrix components in human brain tumor and melanoma cells in respect to variant culture conditions. J Neurooncol 44: 23-33, 1999.

3. Nakada M, Okada Y and Yamashita J: The role of matrix metalloproteinases in glioma invasion. Front Biosci 8: e261-e269, 2003.
4. Lafleur MA, Handsley MM and Edwards DR: Metalloproteinases and their inhibitors in angiogenesis. Expert Rev Mol Med 5: $1-39,2003$.

5. Lakka SS, Gondi CS and Rao JS: Proteases and glioma angiogenesis. Brain Pathol 15: 327-341, 2005.

6. Mook OR, Frederiks WM and van Noorden CJ: The role of gelatinases in colorectal cancer progression and metastasis. Biochim Biophys Acta 1705: 69-89, 2004.

7. Pellikainen JM, Ropponen KM, Kataja VV, Kellokoski JK, Eskelinen MJ and Kosma VM: Expression of matrix metalloproteinase (MMP)-2 and MMP-9 in breast cancer with a special reference to activator protein-2, HER2, and prognosis. Clin Cancer Res 10: 7621-7628, 2004.

8. Talvensaari-Mattila A and Turpeenniemi-Hujanen T: Preoperative serum MMP-9 immunoreactive protein is a prognostic indicator for relapse-free survival in breast carcinoma. Cancer Lett 217: 237-242, 2005.

9. Andersson U, Guo D, Malmer B, Bergenheim AT, Brannstrom T, Hedman $\mathrm{H}$ and Henriksson R: Epidermal growth factor receptor family (EGFR, ErbB2-4) in gliomas and meningiomas. Acta Neuropathol 108: 135-142, 2004.

10. Shepard HM, Jin P, Slamon DJ, Pirot Z and Maneval DC: Herceptin. Handb Exp Pharmacol pp183-219, 2008.

11. Hsieh AC and Moasser MM: Targeting HER proteins in cancer therapy and the role of the non-target HER3. Br J Cancer 97: 453-457, 2007.

12. Higashiyama $S$, Iwabuki $H$, Morimoto $C$, Hieda $M$, Inoue $H$ and Matsushita N: Membrane-anchored growth factors, the epidermal growth factor family: beyond receptor ligands. Cancer Sci 99: 214-220, 2008.

13. Higashiyama S: Metalloproteinase-mediated shedding of heparinbinding EGF-like growth factor and its pathophysiological roles. Protein Pept Lett 11: 443-450, 2004.

14. Chetty C, Bhoopathi P, Joseph P, Chittivelu S, Rao JS and Lakka SS: Adenovirus-mediated siRNA against MMP-2 suppresses tumor growth and lung metastasis in mice. Mol Cancer Ther 5: 2289-2299, 2006.

15. Mohan PM, Chintala SK, Mohanam S, Gladson CL, Kim ES, Gokaslan ZL, Lakka SS, Roth JA, Fang B, Sawaya R, Kyritsis AP and Rao JS: Adenovirus-mediated delivery of antisense gene to urokinase-type plasminogen activator receptor suppresses glioma invasion and tumor growth. Cancer Res 59: 3369-3373, 1999.

16. Mohanam S, Chandrasekar N, Yanamandra N, Khawar S, Mirza F, Dinh DH, Olivero WC and Rao JS: Modulation of invasive properties of human glioblastoma cells stably expressing amino-terminal fragment of urokinase-type plasminogen activator. Oncogene 21: 7824-7830, 2002.

17. Hermisson M and Weller M: NF-kappaB-independent actions of sulfasalazine dissociate the CD95L- and Apo2L/TRAIL-dependent death signaling pathways in human malignant glioma cells. Cell Death Differ 10: 1078-1089, 2003.

18. Cho SG and Choi EJ: Apoptotic signaling pathways: caspases and stress-activated protein kinases. J Biochem Mol Biol 35: 24-27, 2002.

19. Fillmore HL, van Meter TE and Broaddus WC: Membrane-type matrix metalloproteinases (MT-MMPs): expression and function during glioma invasion. J Neurooncol 53: 187-202, 2001.

20. Liang J, Jiang D, Griffith J, Yu S, Fan J, Zhao X, Bucala R and Noble PW: CD44 is a negative regulator of acute pulmonary inflammation and lipopolysaccharide-TLR signaling in mouse macrophages. J Immunol 178: 2469-2475, 2007.

21. Fan Y, Dutta J, Gupta N, Fan G and Gelinas C: Regulation of programmed cell death by NF-kappaB and its role in tumorigenesis and therapy. Adv Exp Med Biol 615: 223-250, 2008.

22. Wang Z, Cao N, Nantajit D, Fan M, Liu Y and Li JJ: MKP-1 represses JNK-mediated apoptosis via NF-kappa B regulation. J Biol Chem 283: 21011-21023, 2008.

23. Bao Q and Shi Y: Apoptosome: a platform for the activation of initiator caspases. Cell Death Differ 14: 56-65, 2007.

24. Kargiotis O, Chetty C, Gondi CS, Tsung AJ, Dinh DH, Gujrati M, Lakka SS, Kyritsis AP and Rao JS: Adenovirus-mediated transfer of siRNA against MMP-2 mRNA results in impaired invasion and tumor-induced angiogenesis, induces apoptosis in vitro and inhibits tumor growth in vivo in glioblastoma. Oncogene 27: 4830-4840, 2008.

25. Sanderson MP, Dempsey PJ and Dunbar AJ: Control of ErbB signaling through metalloprotease mediated ectodomain shedding of EGF-like factors. Growth Factors 24: 121-136, 2006.

26. Luo J: Role of matrix metalloproteinase-2 in ethanol-induced invasion by breast cancer cells. J Gastroenterol Hepatol 21 (Suppl 3): S65-S68, 2006. 